\title{
Thoracoscopic mediastinal repositioning for postpneumonectomy syndrome
}

\author{
Michael F. Reed, MD, and Jaime D. Lewis, MD, Cincinnati, Ohio
}

$\mathrm{P}$ ostpneumonectomy syndrome is characterized by excessive mediastinal rotation and shift resulting in main bronchial obstruction. ${ }^{1}$ More common in children, women, and those undergoing their first thoracic procedure, this entity of symptomatic airway compression may occur after right or left pneumonectomy. ${ }^{2,3}$ Symptoms including stridor, dyspnea, and recurrent respiratory infections should be investigated by computed tomography (CT) and bronchoscopy. Patients found to have postpneumonectomy syndrome should undergo prompt therapy to alleviate their symptoms and prevent irreversible malacic changes of the airway. Here we report thoracoscopic mediastinal repositioning with intrapleural prostheses in a young woman in whom dyspnea and stridor developed after a right pneumonectomy.

\section{Clinical Summary}

A 20-year-old woman with Swyer-James (MacLeod) syndrome underwent thoracoscopic right pneumonectomy at 19 years of age. Her initial postoperative course was uneventful. However, mild dyspnea and stridor developed approximately 3 months after surgery. Over the ensuing 4 months, her symptoms of airway obstruction progressed. A CT scan of the chest demonstrated mediastinal shift to the right and narrowing of the left main bronchus as it crossed the descending aorta (Figure 1). Pulmonary function testing showed an obstructive defect with a forced expiratory volume in 1 second of $1.52 \mathrm{~L}$ (42\% predicted) (Figure 2). Bronchoscopy verified left main bronchial narrowing.

Mediastinal repositioning was performed through a thoracoscopic approach. Two prior thoracoscopy port incisions were opened: a 12-mm site in the sixth intercostal space at the anterior axillary line and a 12-mm site in the eighth intercostal space at the posterior axillary line. Adhesions were lysed to facilitate replacement of the mediastinum centrally. A 6-cm access thoracotomy in the fourth intercostal space in the axilla was then reopened. At no time was rib spreading performed. Two saline breast implants were then placed through the access thoracotomy into the right

From the Division of Thoracic Surgery, Department of Surgery, University of Cincinnati College of Medicine, Cincinnati, $\mathrm{OH}$.

Received for publication July 6, 2006; accepted for publication Aug 8, 2006.

Address for reprints: Michael F. Reed, MD, University of Cincinnati Medical Center, Department of Surgery, Division of Thoracic Surgery, 231 Albert B. Sabin Way, ML 0558, Cincinnati, OH 45367-0558 (E-mail: michael.reed@uc.edu)

J Thorac Cardiovasc Surg 2007;133:264-5

$0022-5223 / \$ 32.00$

Copyright @ 2007 by The American Association for Thoracic Surgery doi:10.1016/j.jtcvs.2006.08.037 hemithorax. As the left main bronchus was visualized with the flexible fiberoptic bronchoscopy, 1 implant (apical) was filled with $450 \mathrm{~mL}$ of saline and 1 implant (basilar) was filled with $700 \mathrm{~mL}$ of saline. Improved airway patency was demonstrated bronchoscopically. Her postoperative course was smooth, and she was discharged on the second postoperative day.

At follow-up in 3 months, she had returned to college and regular activities. She experienced neither dyspnea nor stridor. Chest CT demonstrated the mediastinum near midline and improved patency of the left mainstem bronchus (Figure 1). Her forced expiratory volume in 1 second increased to $2.23 \mathrm{~L}(62 \%$ predicted) (Figure 2).

\section{Discussion}

Postpneumonectomy syndrome is a rare complication after pneumonectomy. Several techniques have been described in an attempt to alleviate the symptoms and prevent or correct the malacic changes that may develop over time. ${ }^{4}$ Methods include insufflation of air into the pleural space, insertion of Lucite balls into the empty thoracic cavity, and lysis of adhesions with phrenectomy on the postpneumonectomy side. In the largest series of patients, Grillo and colleagues ${ }^{2}$ described repositioning with lysis of adhesions to allow the mediastinum to return to its former position, suturing the pericardium to the posterior sternum, and placing intrapleural prostheses in the majority of patients. Endobronchial stent placement has also been used. ${ }^{4}$ Repositioning and placement of intrapleural prostheses seem to be superior to other methods of correction but require a second thoracotomy.
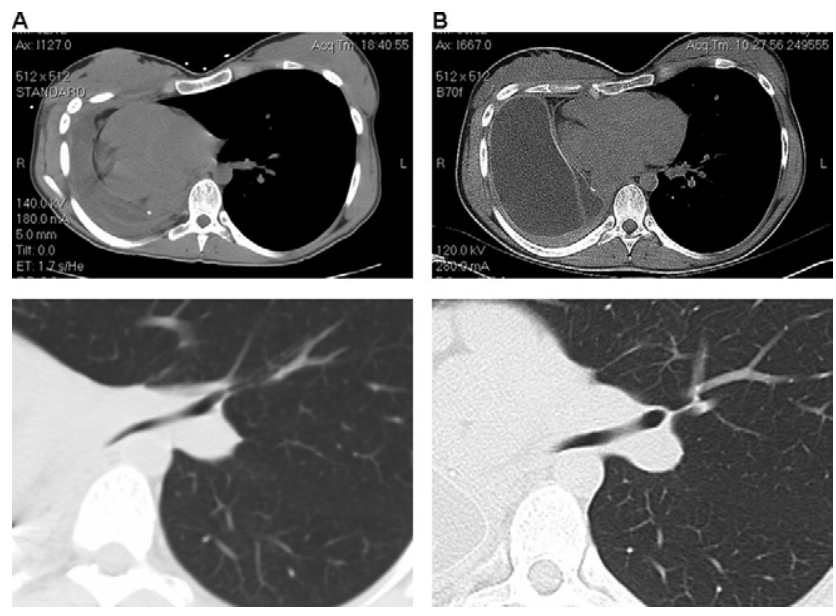

Figure 1. Chest CT. A, Postpneumonectomy syndrome with rightward mediastinal shift and left main bronchial compression. B, Postoperative result with 2 saline implants in the right hemithorax, the mediastinum near midline, and improved left main bronchial diameter. 


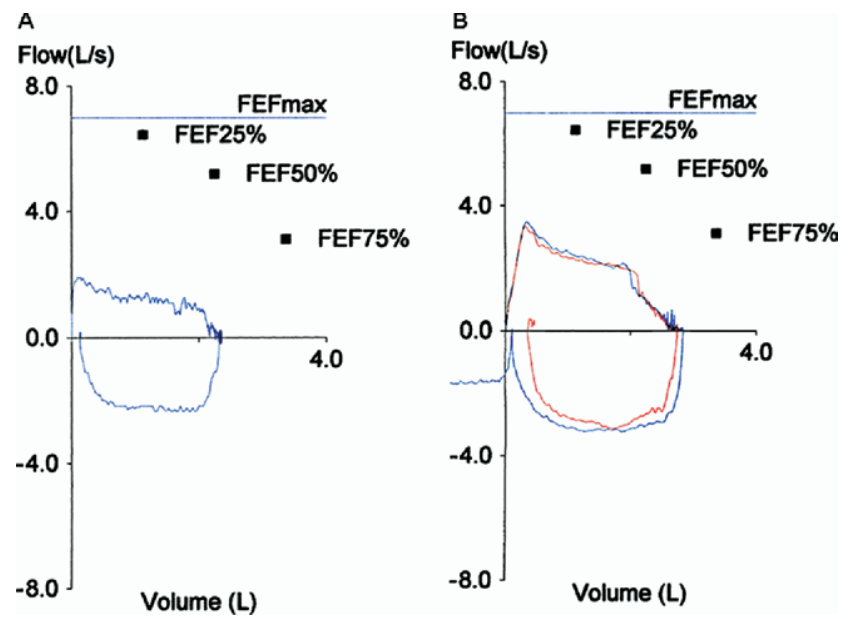

Figure 2. Pulmonary function testing. A, Preoperative flow-volume loop showing significant obstruction. B, Postoperative flow-volume loop demonstrating decreased obstruction. FEF, Forced expiratory flow.

Adoption of less-invasive surgical techniques may improve outcomes by decreasing morbidity, shortening hospital stay, and providing a more desirable cosmetic result, particularly when comparing thoracotomy with thoracoscopy. However, it remains imperative that the fundamental principles that apply to an open approach are followed when performing minimally invasive surgery. In the case of mediastinal repositioning, key points are thorough lysis of adhesions to permit mobilization of the mediastinal structures, placement of prostheses to maintain mediastinal position, and intraoperative bronchoscopic verification of improved airway patency. Here we strictly adhered to these principles. We demonstrated that it is feasible to perform mediastinal repositioning for postpneumonectomy syndrome by using a minimally invasive approach.

\section{References}

1. Adams HD, Junod F, Aberdeen E, Johnson J. Severe airway obstruction caused by mediastinal displacement after right pneumonectomy in a child. A case report. J Thorac Cardiovasc Surg. 1972;63: 534-9.

2. Grillo HC, Shepard JA, Mathisen DJ, Kanarek DJ. Postpneumonectomy syndrome: diagnosis, management, and results. Ann Thorac Surg. 1992; 54:638-50; discussion 650-1.

3. Shamji FM, Deslauriers J, Daniel TM, et al. Postpneumonectomy syndrome with an ipsilateral aortic arch after left pneumonectomy. Ann Thorac Surg. 1996;62:1627-31.

4. Mehran RJ, Deslauriers J. Late complications. Postpneumonectomy syndrome. Chest Surg Clin North Am. 1999;9:655-73.

\section{Primary pulmonary malignant schwannoma with extension to the tracheal carina}

Mika Uchiyama, MD, ${ }^{\text {a }}$ Yoshie Shimoyama, MD, ${ }^{\mathrm{b}}$ Noriyasu Usami, MD, ${ }^{\text {a }}$

Simon Ito, MD, ${ }^{a}$ Ayuko Yasuda, MD, ${ }^{a}$ Koji Kawaguchi, MD, and

Kohei Yokoi, MD, ${ }^{\text {a Nagoya, Japan }}$

$\mathrm{P}$ rimary pulmonary malignant schwannomas are extremely rare, and only 11 case reports of this tumor are available. ${ }^{1}$ All cases were treated with a relatively simple surgical procedure, such as pneumonectomy, lobectomy, or enucleation, but their clinicopathologic appearances have not

aFrom the Division of Thoracic Surgery, Department of Surgery, Nagoya University Graduate School of Medicine, ${ }^{\text {a }}$ and the Department of Pathology and Clinical Laboratories, Nagoya University Hospital, ${ }^{\mathrm{b}}$ Nagoya, Japan.

Received for publication July 21, 2006; accepted for publication Sept 5, 2006.

Address for reprints: Mika Uchiyama, MD, Division of Thoracic Surgery, Department of Surgery, Nagoya University Graduate School of Medicine, 65 Tsurumai-cho, Showa-ku, Nagoya 466-8550, Japan (E-mail: takepon@ med.nagoya-u.ac.jp).

J Thorac Cardiovasc Surg 2007;133:265-7

$0022-5223 / \$ 32.00$

Copyright (C) 2007 by The American Association for Thoracic Surgery doi:10.1016/j.jtcvs.2006.09.004 been well clarified. We present a patient with this tumor, which originated in the right upper lobe of the lung and involved the main-

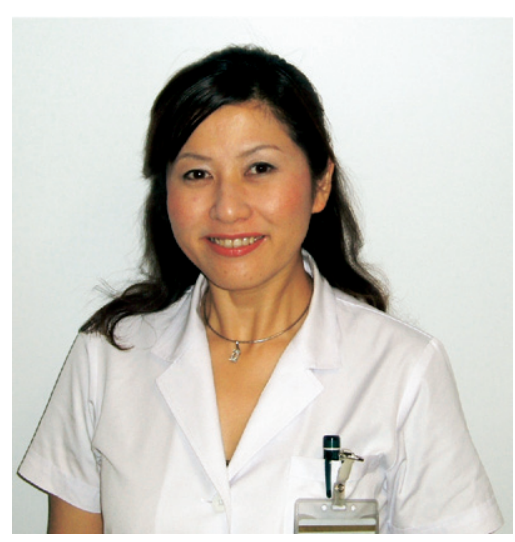

Dr Uchiyama stem bronchus and tracheal carina. For complete removal of the tumor, we performed right upper lobectomy of the lung using extended wedge resection of the tracheal carina, followed by anastomosis of the right intermediate trunk and the distal trachea.

\section{Clinical Summary}

A 57-year-old woman was admitted to our hospital with a 5-month history of worsening cough and dyspnea. There were no abnormal findings on physical and laboratory examinations. Chest x-ray films and computed tomographic scans revealed an apical rightsided large mass measuring $8.4 \times 6.0 \mathrm{~cm}$, which was adjacent to 